\title{
CRITÉRIOS DE AVALIAÇÃO EM ENFERMAGEM
}

\author{
F. G. Abdellah (*)
}

A pesquisa em enfermagem cai numa área onde critérios de avaliação ainda estão para ser desenvolvidos. Considerável esforço terá que ser canalizado para essa finalidade. O propósito deste trabalho é sugerir meios para desenvolver tais critérios. Critério de avaliação é um valor na variável dependente a ser predita, que pode ser usado como base no julgamento para medir o efeito do estímulo aplicado ao objeto da experimentação (1) .

Os critérios de avaliação são pairticularmentee necessários à elaboração de uma pesquisa experimental em enfermagem. No presente contamos com estudos volumosos que mostram o que a enfermeira faz; possuímos, porém, pouca informação sobre o efeito da ação da enfermagem na evolução do estado do paciente (2).

Os critérios de avaliação podem nos ajudar de diversas formas. Vejamos algumas:

Modificação na prática de enfermagem para melhorar o cuidado do paciente. Um estudo mostrou que os pacientes relatavam poucas omissões no cuidado de enfermagem quando havia alta proporção de horas de pessoal profissional (enfermeiras) disponível (3). Neste caso o estímulo aplicado foi uma quantidade adicional de horas de enfermagem profissional. O efeito (variável dependente) mensurado foi a satisfação do paciente, expresso como um valor da variável dependente.

Da mesma forma pode-se testar experimentalmente os efeitos da admissão dos pacientes em uma unidade de auto-cuidado (self care), onde aprendem a tomar sua medicação, fazer tratamentos

(*) Nursing Research 10 (1):21-26, Winter 1961. Tradução de Circe de Mello Ribeiro. 
simples e selecionar sua dieta. O efeito específico a ser medido seria a readmissão ou não do paciente na clínica, consultório médico ou no hospital. A readmissão, neste caso, poderia estar relacionada com uma necessidade residual de cuidados de enfermagem que deveria ter sido satisfeita durante a hospitalização prévia. Por exemplo: se um paciente cardíaco não participa ativamente na sua terapêutica ele pode desencorajar-se e não respeitar a dieta ou não tomar a medicação.

Avaliação do efeito da ação da enfermagem na evolução do estado do paciente. Se o estímulo aplicado a um grupo experimental de pacientes cirúrgicos fosse uma quantidade adicional de cuidado intensivo de enfermagem, a variável dependente, ou o efeito previsto poderia ser medido e expresso como um valor a variável dependente (critério de avaliação). Os efeitos previstos podem incluir: redução nas complicações cirúrgicas tais como infecção da ferida operatória, estabilização do pulso e pressão, aumento da capacidade do paciente de cuidar de si próprio.

Definição e identificação da ciência da enfermagem. Um corpo de conhecimentos científicos quando definidos deverá conter uma tipologia de problemas de enfermagem e uma tipologia dos procedimentos da prática da enfermagem baseados nos princípios de uma ciência de enfermagem ou de ciências correlatas (4'). Disto pode resultar um curriculum centralizado no paciente (estímulo) que pode ser testado experimentalmente. O efeito sobre um grupo de estudantes de enfermagem poderia então ser medido e expresso como um valor da variável dependente (critério de avaliação).

Muitos outros exemplos podem ser dados para ilustrar o uso de critérios de avaliação na prática de enfermagem. Certamente podem nos ajudar a responder perguntas como estas: o que as enfermeiras deveriam estar fazendo ,utilizando de preferência fatos, ao invés de determinar o que estão fazendo, segundo um conhecimento empirico. Enfermagem não é a única profissão a qual faltam medidas específicas da avaliação da prática.

\section{TIPOS DE CRITÉRIOS DE AVALIAÇÃO EM ENFERMAGEM}

A premissa básica é que critérios de avaliação da prática da enfermagem devem derivar das variáveis dependentes, as quais indicam os efeitos dessa prática no cuidado do paciente. Tais medidas podem ser classificadas em dois grupos: $1 .^{\circ}$ - aquelas relacionadas com as reações fisiológicas do doente à moléstia; 2 . $^{\circ}$ aquelas relacionadas às suas necessidades psico-sociais. 
Critérios de avaliação relacionados com as necessidades fisiológicas dos pacientes são medidas visíveis que podem ser identificadas. Exemplos que ajudam a retratar as respostas dos pacientes à moléstia, em termos de uma escala de valores, são: temperatura, pulso, respiração, pressão arterial, condições da pele, estado de nutrição (p. ex. peso), tonus muscular, manutenção do mecanismo e funções da regulação do corpo humano, incluindo funções sensoriais (reação à dor, sono, inquietação, eliminação); grau de mobilidade.

Critérios de avaliação de reações de origem fisiológica podem, frequentemente, ser determinados com a ajuda de instrumentos como termômetro, esfigmomanômetro, diapasão ou por observação direta. O instrumento permite uma escala de valores a ser usada no critério de avaliação que será mais preciso do que aproximado. Isto ajuda o investigador a fazer previsões sobre o critério de avaliação $\mathrm{em}$ termos de frequência e probabilidade.

\section{DIFICULDADE EM IDENTIFICAR CRITÉRIOS}

Se critérios de avaliação podem ajudar a enfermagem, e consequentemente, melhorar o cuidado de enfermagem, porque esses critérios não foram identificados? Há muitas razões. Discutirei apenas algumas.

As próprias enfermeiras não conseguem chegar a um acordo quanto aos critérios para a avaliação da eficiência do cuidado de enfermagem. Um corpo de conhecimentos científios que seja exclusivo da enfermagem está ainda por ser identificado, a fim de oferecer uma abordagem teórica com a qual á prática da enfermagem possa ser relacionada. Os critérios de avaliação na enfemagem; diversamente da pesquisa controlada em laboratório, na qual o organismo estudado está num meio controlado (um tubo de ensaio ou gaiola), devem ser feitos no ambiente complexo do paciente. Como há muitas variáveis na situação física e psicológica, torna-se mais difícil isolar variáveis específicas.

As dificuldades em identificar critérios de avaliação em enfermagem têm levado muitas investigações para áreas onde há maiores facilidades para pesquisas. Para ilustrar: o estudo sobre o que faz a enfermcira, quanto tempo dispende no cuidado ao paciente, pode apenas nos dar conhecimentos empíricos (5). Este conhecimento tem valor porque indica áreas de problemas que devem ser estudados mais profundamente. A atuação de enfermeira deve, fundamentalmente, ser avaliada de acordo com os efeitos (critérios de avaliação) da prática de enfermagem sobre os pacientes. 
Da mesma forma, os estudos sobre o papel da enfermeira têm valor porque indicam uma direção para a enfermagem como profissão (6) .

Estes estudos são valiosos, porém, têm significado pouco decisivo se não houver um critério de avaliação para medir seus efeitos sobre o cuidado ao paciente. Assim, podemos supor, falsamente, que estamos melhorando o cuidado de enfermagem sem o conhecimento do efeito desse cuidado sobre o paciente. Talvez estejamos satisfazendo a necessidade dos hospitais, dos médicos, das enfermeiras, mas não dos pacientes.

A falta de critérios de avaliação em enfermagem limita parcialmente a enfermeira quando presta cuidados de enfemagem. Sua atuação é sobretudo por ensaio e erro, ao invés de ter como base um procedimento cientificamente provado como eficiente.

Critérios de avaliação relacionados às necessidades psico-sociais são mais difíceis de determinar porque a avaliação é mais aproximada do que precisa. Critérios preliminares de avaliação são muitas vezəs exprcssos como componentes essenciais resultantes de um efeito, os quais podem ser traduzidos em valor da variável dependente ou resposta, como por exemplo:

a) a comureensão do paciente quanto à sua condição e o seu ajustamento às reações fisiológicas do próprio organismo à dotnça:

b) habilidade em verbalizar reações emocionais;

c) habilidade em reconhecer sentimentos positivos e negativos, sem ansiedade ou resistência;

d) habilidade em aceitar e ajustar-se às próprias limitações;

e) auto-confiança;

f) senso de independência dos outros, sua atividade para desenvolver habilidade no cuidado próprio;

g) percepção do cuidado de enfermagem que recebe.

Tais critérios de avaliação são difíceis de observar. Não há instrumento para dar uma medida absoluta da aceitação e compreensão do paciente quanto às suas condições. Os instrumentos que têm sido desenvolvidos para avaliar a satisfação do paciente quanto ao cuidado que recebem são baseados em um valor de variável de- 
pendente que reflete uma composição de diversas variáveis (7). Essts valores representam padrões aos quais o paciente, indivicualmenie, $i$ comparado.

\section{Como sclecionar medidas de avaliação}

Para validade de um critério de avaliação, o qual deve ser expresso ccmo um valor da variável dependente, ele deve satisfazer duas condições - validade e segurança (8).

A validade é evidenciada na extensão em que várias medidas ou valores de eficiência são correlacionados com o critério de avaliação. Por exemplo, num estudo de prematuros o peso ganho é uma medida válida de avaliação se correlacionada com outras medidas, tais como - bom padrão de comportamento na alimentação e períodos de sono sem interrupção. Apenas aumento de peso, sem os corolários positivos, não pode ser um critério válido.

Se alguém tiver que determinar a validade do critério de avaliação da capacidade de um paciente de cuidar de si próprio, essa medida terá que estar bem correlacionada com outras, tais como: capacidade do paciente de aceitar e ajustar-se às suas limitações, habilidade em usar outros recursos e tornar-se independente dos outros. Assim, é muito improvável que um critério de avaliação rálido esteja correlacionado com medidas negativas.

Se o critério é válido deve satisfazer também as especificações ce relevância e adequação. Podemos selecionar um critério relativo à mobilidade do paciente, por ex.: habilidade do paciente em ir a fila do refeitório e selecionar sua alimentação. Pode ser este um bom critério para alguns pacientes, mas não será relevante ou adequado para um paciente que tenha bloqueio mental quanto a alimentação, mesmo que ele seja fisicamente capaz de ir ao refeitório. Neste caso, não será um critério válido.

Para ser válido, o critério deve ser discriminativo. Uma graouação de medidas adequadas pode, quase sempre, aumentar a correlação entre outras medidas de sucesso e o critério de avaliação. Quanto mais discriminativo for um critério de avaliação, mais váliço será o resultado da variável dependente na mensuração da eficiência de uma variável independente (o estímulo aplicado que resultará em um efeito).

O pesquisador pode selecionar critérios que sejam válidos, porém, alguns são menos característicos por causa da existência de obstáculos nem sempre aparentes. Po rex.: se for usada a mobilidade do paciente como critério para medir o efeito do cuidado de en- 
fermagem no seu progresso, vârios graus de mobilidade podem ser selecionados. A eficiência pode ser medida em termos da distância coberta numa superfície plena ou quando obstáculos específicos são introduzidos.

Uma das medidas de eficiência pode ser a capacidade do paciente ir até o restaurante, introduzindo como obstáculos escadas e cruzamentos de ruas, ou sua habilidade em ir ao banheiro com alguns obstáculos colocados no caminho. Quais são as medidas mais características do progresso do paciente? Medidas errôneas podem ser selecionadas, se não houver, previamente, uma verificação da situação e identificação de todas as dificuldades. A mobilidade pode ser usada como um critério válido, porém a medida da mobilidade do paciente 20 ir ao banheiro, quando não estejam presentes os obstáculos, escada e cruzamento de rua, será menos característica que a medida do progresso do paciente quando vai para a fila do restaurante.

Segurança é a segunda especificação importante de um critério de avaliação. Um critério de avaliação será de confiança se medir de forma consistente o que se propõe a medir. Por ex.: em um estudo sobre omissões no cuidado de enfermagem e os seus efeitos na satisfação do paciente, foi encontrado que, durante o teste piloto da lista de verificação usada como instrumento de avaliação, os pacientes respondiam mais precisa e consistentemente quando completavam a lista no momento em que as omissões ocorriam, do que mais tarde, durante o período de hospitalização ou após a alta (9).

Um. estudo de caso

Gostaria de usar o estudo sobre o cuidado progressivo do paciente para ilustrar as formas pelas quais os critérios de avaliação podem ser desenvolvidos. A equipe de pesqusa formada de médicos, enfermeiras, um bioestatístico, administradores hospitalares, um psicologo, um sociólogo, um engenheiro industrial, arquitetos, especialistas em equipamento, nutricionistas, uma bibliotecária e um analista de custos, concordou com uma definição operacional de cuidado progressivo do paciente (CPP).Cuizado progressivo do paciente foi definido como a organização de facilidades, serviços e pessoal, tendo como centro as necescidades, médicas e de enfermagem dos paciente. O cuidado progressivo inclui cinco e'ementos: cuidado intensivo, cuidado intermeciário, auto-cuidado, cuidado a longo termo e cuidado no lar. Um hospital local de 200 leitos que estava em processo de transição para o sistema de cuidado progressivo foi escolhido para o estudo. Era o 
único hospital num raio de 10 milhas. O Administrador e o Conselho de Administração, tendo que enfrentar o problema da escassez de pessoal de enfermagem e uma longa lista de espera de pacientes, decidiram fazer uma experiência, reorganizando as facilidades do hospital.

Todos os pacientes em estado crítico foram reunidos numa unidade de cuidado intensivo, indepentemente de diagnóstico, sexo ou situação econômica. Um grupo selecionado de pacientes foi colocado numa unidade de auto-cuidado. Estes pacientes eram todos fisicamente capazes de cuidar de si próprios, porém necessitavam de serviços hospitalares como Raio X, laboratório ou orientação para aprenderem a viver com suas limitações. Os pacientes com molestias crônicas ou fraturas, que requeriam hospitalização por mais de 30 dias, forain colocados em uma unidade de cuidado a longo termo. Todos os demais que não se encaixavam nas categorias acima foram colocados em tres unidades de cuidado intermediário.

A tarefa da equipe de pesquisa era estudar esta situação hospitalar para determinar quais os fatores, se havia algum, que, afetando a prática da medicina, enfermagem e nutrição, e os custos e padrões de recuperação de pacientes, pudessem ser atribuídos ao sistema do cuidado progressivo do paciente.

O CPP é um fenômeno constituído de muitas variáveis, resultando em efeitos múltiplos e que aumentam a complexidade da conceitualização. Ao lidar com tão amplo fenômeno, frequentemente é necessário fazer antes um levantamento para determinar as diferencas que poderão ser atribuídas ao CPP. Estas diferenças devem ser posteriormente examinadas por meio de um estudo comparativo causal para identificação dos fatores condicionantes (critérios de avaliação) relacionados ao CPP. Uma vez identificados os critérios de avaliação estes poderiam ser intensivamente analisados, aperfeiçoados e submetidos à experimentação. Essa é a sequência comum nas abordagens de pesquisa.

\section{Identificação dos componentes mais importantes}

Durante a fase inicial de estudo cada disciplina representada na equipe de pesquisa tinha que preparar uma descrição da situação de um ano antes e um ano após o início do programa CPP. Isto é chamado às vezes de experimentação retrospectiva e é útil em situação de mudanças, a fim de evitar uma experimentação prévia.

$\mathrm{O}$ arquiteto usou as plantas físicas originais para esboçar os planos feitos. O administrador hospitalar, o médico e a nutricio- 
nista revisaram as práticas anteriores e descreveram aquelas que nas suas áreas deveriam ser introduzidas em consequência do CPP. $O$ analista de custos preparou novas bases para estimativa de custos, baseado mais nos serviços aos pacientes do que no tipo de serviços ou facilidades físicas.

$\mathrm{Na}$ enfermagem, a enfermeira e o bioestatístico obtiveram folhas de controle de tempo, computaram as horas de cuidado de enfermagem por paciente em cada unidade, antes e depois do início do CPP. O bioestatístico calculou também a média de "turn-over" cie cada categoria de pessoal de enfermagem, antes e depois. Foram efetuados estudos sobre utilização das enfermeiras nas unidades, para determinar os tipos de procedimentos de enfermagem que estavarn sendo providos. Foram feitos estudos sobre a satisfação do pessoal $\epsilon$ dos pacientes a fim de deteminar a atitude em relação ao CPP.

Foram feitas entrevistas com pacientes, médicos e enfermeiras, com o fim de determinar sua percepção e apreciação quanto ao CPP. As características das enfermeiras foram estudadas para determinar a razão pela qual haviam sido selecionadas para determinadas unidades.

Após alguns meses de estudo intensivo dessa situação foram isoladas os principais componentes que compunham o fenômeno CPP. Se pudessem ser descritos numa série de itens dos quais as hipóteses forem extraídas e atribuídas ao CPP, e mais tarde testadas para validade e segurança, poderiam servir como variáveis dependentes para medir os efeitos do CPP. O valor atribuido a cada variável depedente poderia ser traduzido como critério de avaliação.

Seguem os cinco componentes mais importantes (critério de avaliação antes do teste) isolados como resultantes da introdução do CPP e os itens dos quais podem ser levantadas as hipóteses:

Componente n. ${ }^{\circ} 1$ - Mais rápida recuperação do paciente.

Itens 1.1 - O fator tempo, relacionado à rapidez com que os procedimentos médicos podem ser executados, tem efeito direto na recuperação do paciente.

1.2 - O fator tempo, relacionado à rapidez com que os procedimentos de enfermagem podem ser executados, tem efeito direto na recuperação do paciente.

1.3 - As complicações relacionadas com o cuidado do paciente são reduzidas (por ex.: relatórios de incidentes, infiltração por EV). 
1.4 - Redução das visitas médicas devido ao alto nível do cuidado ao paciente e diminuição de complicações.

1.5 - Redução do tempo de permanência com a introdução de um programa de CPP.

1.6 - O paciente é capaz de retornar ao lar e ao trabalho mais rapidamente.

Componente n.o 2 - Aceitação do cuidado progressivo pelo paciente, família, comunidade e pessoal hospitalar, após a introdução do programa.

2.1 - As relações médico, paciente e família melhoraram.

2.2 - Houve melhora nas relações entre os departamentos médicos e a administração do hospital.

2.3 - As relações enfermeira-médico melhoraram.

2.4 - As relações enfermeira-paciente e familiares melhoraram.

2.5 - Os pacientes sentem-se mais satisfeitos.

2.6 - Aumentou a satisfação das enfermeiras com suas condições de trabalho.

2.7 - Os pacientes nas unidades de cuidados intensivos dão maior importância ao cuidado médico e de enfermagem do que ao seu ambiente físico.

Componente n.o 3 - Redução no custo para o paciente, família e hospital.

3.1 - E mais econômico para o paciente, família e instituição ter programas de CPP do que o tipo de serviço usualmente encontrado nos hospitais.

3.2 - As unidades de auto-cuidado podem ser montadas com equipamento doméstico, com maior redução de custo que apresenta o equipamento hospitalar.

3.3 - Os serviços de pessoal podem ser providos na unidade de auto-cuidado a custo reduzido.

3.4 - O equipamento pode ser concentrado em áreas especificas com redução de custos na operação do hospital. Por ex.: Rede de oxigenıo apenas nas unidades de cuidados intensivos ao enves de espelhá-las por todo 0 hospital. 
Componente n. ${ }^{\circ} 4$ - Utilização efetiva das unidades de CPP por: tipo de paciente, médico e enfermeira.

4.1 - Os pacientes médico-cirúrgicos e os emocionalmente perturbados podem ser cuidados nas unidades de CPP.

4.2 - A unidade de auto-cuidado poderá ser utilizada para pacientes convalescentes e não limitada apenas aos pacientes em fase de diagnóstico.

4.3 - Os padrões mínimos e ótimos de pessoal de enfermagem podem ser determinados para cada tipo de unidade do CPP, com base nas necessidades médicas e de enfermagem dos pacientes.

4.4 - As enfermeiras nas unidades de cuidado intensivo e auto-cuidado têm mais tempo para os paciente.

4.5 - O CPP permite uma classificação eficiente dos pacientes baseada em suas necessidades individuais.

4.6 - As unidades de cuidado intensivo devem ser providas com uma enfermeira e um atendente para cada seis pacientes (em cada período).

Componente n. 5 - Planta física funcional.

5.1 - Os futuros planejamentos dos hospitais devem inciuir áreas para cuidado intensivo e para auto-cuidado.

5.2 - As áreas para auto-cuidado devem ser separadas da planta principal do hospital por um corredor de conexão.

5.3 - As áreas de cuidado intensivo devem ser localizadas de forma a permitir fácil movimentação do paciente de uma área para outra.

5.4 - As áreas de cuidado intensivo devem ser montadas de formal que cada uma seja auto-suficiente em termos de equipamento, medicação, material e roupas.

5.5 - Todo o equipamento das áreas de cuidado intensivo deve ser exclusivo e embutido nas paredes.

5.6 - O mobiliário das áreas de cuidado intensivo deve se restringir à cama do paciente.

\section{AVALIAÇÃO DE UM COMPONENTE IMPORTANTE}

Durante a segunda fase foram selecionados pela equipe de pesquisa, para estudo intensivo e experimentação, alguns itens es- 
pecíficos dos tres componentes considerados mais importantes: o primeiro relacionado ao custo, o segundo à distribuição funcional e utilização de leitos por unidade, com base nas transferências de pacientes, e o terceiro relacionado à utilização efetiva das unidades do CPP por tipo de paciente, médico e enfermeira.

Foi escolhido para mensuração o ítem específico (4.3) relacionado com o componente utilização, isto é, a convicção de que padrões mínimos e ótimos de pessoal de enfermagem podem ser determinados para cada tipo de unidade CPP baseados nas necessidades médicas e de enfermagem dos pacientes. Para provar isto foram planejados dois estudos. O primeiro, empírico, para determinar, no país, o padrão de cálculo de enfermeiras e as razões de sua variabilidade nos hospitais gerais com um ou mais tipos de unidades CPP. Essa fase do estudo forneceu informação sobre a prática atual dos hospitais com ou sem unidades CPP quanto aos padrões usados e está relatada em outro trabalho (10).

Esse tipo de informação pode ser útil na avaliação da relevância e adequação de um critério de medida. Por exemplo, a análise dos dados mostrou que padrões de cálculo, absolutos, não podem ser estabelecidos para cada unidade. Para um hospital onde haja grande número de neurocirurgias ou de operações cardíacas a céu aberto, o padrão estabelecido para uma enfermaria geral de medicina e cirurgia não seria realístico. Poderia ser necessário a média de uma enfermeira para cada paciente.

O segundo estudo, de natureza exploratória, foi realizado num grande hospital metropolitano objetivando identificar: (a) uma estrutura conceitual da qual pudessem ser extraídos conceitos para os padrões de provimentto de enfermeiras; (b) critérios de avaliação para medir o efeito dos serviços de enfermagem no progresso do paciente; e (c) níveis de habilidades técnicas em enfermagem requeridos para execução dos serviços.

Um problema significativo na execução de pesquisas experimentais e não experimentais em enfermagem é a falta de estrutura conceitual para as pesquisas propostas. Isto se torna particular-. mente importante quando se está tentando identificar critérios específicos de avaliação.

$\mathrm{Na}$ enfermagem, uma estrutura conceitual é uma abordagem teórica ao estudo de problemas cientificamente fundamentados; enfatiza a seleção e a elucidação de seus conceitos. A abordagem teórica pode oferecer recursos para análise dos dados e agrupar os fatos em fundamentos. A proposição de uma teoria (estrutura conceitual) na pesquisa em enfermagem pode auxiliar a identificação clara e precisa dos conhecimentos na área. 
Foi mencionado anteriormente que uma das tarefas imediatas da equipe de pesquisa seria preparar uma estrutura conceitual da qual pudessem derivar conceitos para padrões de cálculo de enfermeiras. Para ilustrar: uma estrutura conceitual é constituída de tres partes:

1 - Definições: as quais devem ser operacionais.

2 - Conceitos (postulados) : idéias abstratas de significação universal que podem, posteriormente, ser traduzidas em princípios.

3 - Deduções: referem-se às conclusões alcançadas através de processo lógico, partindo da elucidação das definições até o estabelecimento de conceitos aceitáveis e visando as hipóteses a serem testadas.

Segue-se um resumo de uma estrutura conceitual da qual poderia emergir um padrão de cálculo de enfermeiras (11). O primeiro passo foi definir operacionalmente os temos dos quais poderiam derivar os padrões de cálculo de enfermeiras.

\section{DEFINIÇŌES}

\section{Cuidado de enfermagem}

1 - Assistência que é prestada a um paciente quando, por alguma razão, ele não pode satisfazer suas próprias necessidades.

2 - Corresponde às habilidades e destrezas do indivíduo que presta assistência.

3 - Deriva de um estudo das necessidades de cuidado de enfermagem apresentadas pelo paciente.

4 -- É dirigida no sentido de tornar o paciente capaz de cuidar de si próprio.

- Necessida ¡ $a_{i}$ pessoal - Uma variedade considerável de condições ótimas, tais como: ar, alimentos, temperatura, integridade física, são requisitos para a sobrevivência. Quando, por alguma razão, qualquer desses requisitos deixa de ser ótimo, diz-se existir um estalo de necessidade.

Habilidade no auto-cuidado - Capacidade do indivíduo de satisfazer suas próprias necessidades.

Estado patológico - Alteração no organismo ou na pessoa, que limita a capacidade de satisfazer suas próprias necessidades, em 
consequência a um ferimento, moléstia, mal formação ou desenvolvimento anormal.

Uma vez consideradas essas definições, o próximo passo foi o de formular os postulados e deduções relacionados com a necessiclade de cuidados de enfermagem.

Necessidade de cuidado de enfermagem

Postula to - Enquanto a capacidade de auto-cuidado (CAC) for desenvolvida e mantida em nível que permita a satisfação das necessidades sem o auxílio de outrem, o cuidado de enfermagem é dispensável. A satisfação de necessidades será uma função da CAC, etc...

Dedução - Satisfação de necessidade de enfermagem, será então, uma função da capacidade de auto-cuidado e do cuidado de enfermagem.

Necessidade de cuidado de reabilitą̧ão

Postulado - Quando, ccmo resultado de uma alteração patológica, a capacidade de auto-cuidado fica reduzida ao mínimo e certas necessidades pessoais não podem ser satisfeitas, surge a necessidade de cuidado de reabilitação.

Dedução - Cuidado de reabilitação está em função da necessidace de cuidado de manutenção, curativo e de recuperação.

Necessidade de cuidado de manutenção

Postulajo - Quando a CAC está diminuída como resultado de uma alteração patológica e o paciente não pode por, si próprio, satisfazer suas necessidades pessoais, assistência lhe deve ser prestada, a fim de aumentar sua CAC na extensão em que as necessidades pessoais possam ser satisfeitas.

Dedução - A necessidade de cuidado de manutenção está em função da reduzida da CAC e das necessidades pessoais não atendidas.

$\mathrm{NAM}=\mathrm{f}(\mathrm{CAC}-) \mathrm{x}(\mathrm{NPP})$

(A Necessidade de Assistência de Manutenção está em função da Capacidade de Auto-Cuidado negativa multiplicada pelas Necessidades Pessoais do Paciente). 
Se um investigador não prepara uma estrutura conceitual para sua pesquisa, corre o risco de basear seu estudo num simples postulado (conceito) ou em hipóteses que não estão de acordo com os conhecimentos já existentes. Um postulado tem significação somente quando ocorre juntamente com outros, em termos válidos, os quais temos razão para acreditar sejam verdade.

Assim, uma estrutura conceitual determina as relações funcionais entre fatos e não é limitada às relações estatísticas. Os levantamentos descritivos são úteis porque ajudam o pesquisador no desenvolvimento de uma estrutura de teoria.

Foram propostas as seguintes hipóteses chaves para serem testadas:

1 - Os serviços de enfermagem devem ser determinados pelas necessidades dos pacientes.

2 - As necessidades do paciente mudam nas 24 horas, daí a necessidade de sua avaliação diária pela enfermeira e pelo médico responsável. (Isto pode ser feito por uma líder de equipe e um interno).

3 - Há quatro necessidades de assistência que podem ser identificadas: cuidado de manutenção, curativo, de reabilitação e preventivo.

4 - Avaliação é um tipo de serviço que é contínuo.

5 - Há tres níveis de habilidades requeridas para o atendimento das nccessidades dos pacientes: de coordenação, técnicas e de custódia (proteção).

Foi estudada uma amostra de pacientes de clínicas médicas e cirúrgicas desde a admissão até a alta. Algumas fases da assistência de enfermagem foram dadas pelo pessoal do hospital. Aspectos do cuidado de enfermagem que não eram dados aos pacientes em estudo foram providos por uma outra enfermeira e pela investigadora da equipe de pesquisa.

O aspecto mais crucial deste estudo, ainda para ser realizado, é identificação das variáveis dependentes válidas e de confiança as quais quando transformadas em valores, podem ser usadas como critério de avaliação para determinar o efeito dos serviços de enfermagem no progresso dos pacientes.

Espera-se que, no tanscorrer da pesquisa, sejam obtidas informações sobre os padrões de cálculo de pessoal (enfermeiras) nos hospitais gerais com e sem unidades CPP. 
O estudo que acaba de ser descrito difere do levantamento descritivo no sentido de que nele foi feita uma tentativa de desenvolver padrões de cálculo de pessoal (enfermeiras) baseado mais na assistência de enfermagem que deveria ser prestada, a fim de atender as necessidades dos pacientes, do que nos serviços já existentes e que estavam em vias de provimento. O levantamento descritivo do CPP descrito anteriormente mostrou que as facilidades para os pacientes haviam mudado, porém, as práticas de enfermagem não. Uma hipótese que precisa ser tratada é que existem práticas de enfermagem que estão sendo omitidas e que são essenciais para o progresso do paciente. Esse progresso deveria ser medido utilizando-se critérios de avaliação.

Desde que tenham sido identificados as funções, níveis de habilidade e critérios de avaliação, devem ser efetuadas experiências com vários níveis de pessial, para determinar quais os grupos que podem desempenhar adequadamente os serviços de enfermagem, baseados nas necessidades dos pacientes. Isto levará à utilização de várias categorias de pessoal de enfermagem (profissional e auxiliar) para determinar a eficiência das práticas de enfemagem na satisfação das necessidades dos pacientes. A eficiência seria medida por critérios de avaliação elaborados durante o trabalho com uma amostra de pacientes.

\section{SUMÁRIO}

Os critérios de avaliação em enfermagem são de crucial importância se quisermos avaliar o efeito da prática da enfermagem na evolução do paciente. A descoberta de critérios de avaliação podem ajudar-nos a elucidar o papel da enfermeira e nos levar à identificação de um corpo de conhecimentos científicos exclusivo da enfermagem.

Os critérios, em enfermagem, podem ser classificados em fisiológicos, psicológicos e sociológicos. Devem satisfazer as especificações de validade, discriminação, adequação e confiança. Podem nos ajudar a medir o progresso do paciente.

\section{REFERENCIAS BIBLIOGRAFICAS}

1. ENGLISH, H. B. \& ENGLISH, A. C. A comprehensive dictionary of psychological and psychoanalytical terms. New York, Longmans, Green and Co., 1958. 
2.HUGHES, E. C. \& HUGHES, H. M. Twenty thousand nurses tell their story. Philadelphia, J. B. Lippincott Co., -958.

3. ABDELLAH, F. G. \& LEVINE, E. Effect if nurse staffing onsatisfactions with nursing care. (Hospital monograph n. ${ }^{\circ}$ 4). Chicago, American Hospital Association, 1958.

4. ABDELLAH, F. G. and others. Patient-centered approaches to nursing. New York, Macmillan Co., 1960.

5. HUGHES, op. cit.

6. HABERSTEIN, R. W. \& CHRIST, E. A. Professionalizers, traditionalizers and utilizers. Columbia, Mo., University of Misssouri, 1955.

7. ABDELLAH \& LEVINE, op. cit.

8. BROWN, A. F. Research in nursing. Philadelphia, W. B. Saunders Co., 1958.

9. ABDELLAH \& LEVINE, op. cit.

10. ABDELLAH, F. G. and others. Nursing patterns vary in progressive care. Mod. Hosp. 95:85-91, aug. 1960.

11. ABDELLAH, F. G. and others. Patient-centerer approaches to nursing. New York, Macmillan Co., 1960. 